\title{
Resuscitative Thoracotomy as a Life-saving Intervention in the Emergency Department
}

\author{
Fatih Doğanay ${ }^{1}$ and Feride Güneç ${ }^{2}$ \\ ${ }^{1}$ Department of Emergency Medicine, Edremit State Hospital, Balkesir, Turkey \\ ${ }^{2}$ Department of Thoracic Surgery, Edremit State Hospital, Balıkesir, Turkey
}

\begin{abstract}
Objective: To investigate the survival rate and the return of spontaneous circulation (ROSC) in patients who underwent the resuscitative thoracotomy in the emergency department (EDRT), and determine the difference in survival rate of the patients who underwent EDRT with and without out of hospital CPR.

Study Design: Observational study.

Place and Duration of Study: Department of Emergency Medicine, Edremit State Hospital, Balıkesir, Turkey between January 2014 and March 2020.

Methodology: Patients who underwent EDRT were included. Post-CPR ROSC success and 6-month survival were determined as the study endpoints. Categorical data were compared using the Chi-square test so expressed as frequency and percentage, and continuous parametric data were expressed as mean \pm standard deviation (SD).

Results: Twenty-seven patients were included in the study. The 6-month survival was $51.9 \%$ for all patients, and ROSC was achieved in $61.1 \%$ of patients requiring CPR. Comparing patients with and without out of hospital CPR, survival was observed in $16.7 \%$ and $80 \%$. ROSC success was observed in $50 \%$ of patients who needed out of hospital CPR, while $83.3 \%$ for the patients with in-hospital cardiac arrest. The survival rate was 33.3\% in gunshot and $80 \%$ in stab-wound injuries. Survival did not occur in patients with blunt trauma.

Conclusion: Performing EDRT increases the survival in penetrating injuries, especially in stab-wounds. Patients gain time for an advanced intervention. Furthermore, EDRT provides life guardians time to assess for organ donation when death is inevitable.
\end{abstract}

Key Words: Blunt trauma, Cardiac arrest, CPR, Emergency thoracotomy, Gunshot injury; Organ donation, Resuscitation, Resuscitative thoracotomy, Stab-wound injury; Trauma resuscitation.

How to cite this article: Doğanay F, Güneç F. Resuscitative Thoracotomy as a Life-saving Intervention in the Emergency Department. J Coll Physicians Surg Pak 2021; 31(06):627-632.

\section{INTRODUCTION}

According to 2019 data from the World Health Organisation (WHO), the second most common cause of death was trauma. ${ }^{1}$ Multidisciplinary trauma hospitals and trauma teams are known to reduce mortality and morbidity in trauma resuscitation. ${ }^{2}$ In recent years, the importance of a focused approach in identifying the underlying cause of trauma with rapid multidisciplinary interventions has been emphasised incardiopulmonary resuscitation (CPR), especially in trauma patients. ${ }^{3}$ MacKenzie et al. compared the mortality results of trauma patients treated in trauma centres and non-trauma centres. They reported that the mortality rate was $25 \%$ lower in trauma centres. ${ }^{4}$

Correspondence to: Dr. Fatih Doganay, Department of Emergency Medicine, Edremit State Hospital, Balıkesir, Turkey

E-mail: drdoganay@gmail.com

Received: March 11, 2021; Revised: April 22, 2021;

Accepted: May 04, 2021

DOI: https://doi.org/10.29271/jcpsp.2021.06.627
Demetrios et al. reported that the second most common cause of in-hospital death was haemorrhagic shock in patients admitted to the hospital after trauma. They also reported that deaths due to haemorrhagic shock usually occurred within the first 6 hours. They observed that mortality rates decreased after bleeding control with early surgical intervention. ${ }^{5}$

Levitsky et al. in the Vietnam War, reported that emergency thoracotomy was life-saving in soldiers with gunshot wounds, and recommended that thoracotomy could be performed in the Emergency Department in patients who had signs of life in gunshot injuries but do not have a pulse. ${ }^{6,7}$ Over the time, the life-saving effect of the emergency department resuscitative thoracotomy (EDRT) has been proven, and early surgical intervention in the Emergency Department (ED) is on the way to be the main resuscitative approach in trauma patients. It is clear that the standard CPR approach is insufficient for severe lifethreatening situations, such as tension pneumothorax, cardiac injury, major vascular injury and cardiac tamponade. ${ }^{8}$ Both the importance of the trauma team in post-traumatic resuscitation and the EDRT in life-threatening thoracic injuries have been emphasised in recentyears. ${ }^{9}$ 
The aim of the study was to investigate the survival rate of patients who underwent EDRT in a secondary hospital. The secondary aim was to investigate survival rate in patients and determine the ROSC success due to EDRT in patients who neededCPR.

\section{METHODOLOGY}

The study was planned as an observational retrospective study, with analysis of the data from patients who underwent EDRT at Edremit State Hospital, Balıkesir, Turkey between January, 2014 and March, 2020. The patients included in the study were sourced from the Hospital Information Management System (HBYS) database by scanning the diagnosis codes that contained thoracotomy. The analysed data were collected by checking HBYS database. The 6-month survival status of patients who were discharged or transferred to another hospital was obtained from their follow-up files or phone numbers registered in the HBYS.

The ambulanceservicenotified theED by a phonecall when transporting a patient with life-threatening conditions, such as injuries from shooting, stabbing, traffic accident and falling from dangerous heights or for cases requiring CPR. This phone call provided time for the ED team to prepare intervention in a timely and appropriate manner. During the period of inclusion in the study, there was no cardiovascular surgeon (CVS) in the hospital where the study was conducted. Thoracotomies were performed in the ED resuscitation room by an experienced thoracic surgeon in the presence of an emergency physician. After primary lifesaving repair, the remainder of the operation was continued in the operating room for patients with ROSC. Patients who had ROSC and needed cardiovascular surgery after primary surgical intervention were referred.

Age, gender, mechanism of trauma, localisation of injury, out of hospital CPR necessity, in-hospital CPR necessity, Glasgow coma score (GCS) upon arrival at the hospital, whether patients in necessity of out of hospital CPR could be provided with ROSC, referral requirement, 6-month survival, 6-month neurological performance and organ donation status information were obtained via the HBYS database.

Patients were divided into 3 groups, according to the mechanism of trauma. These groups were identified as stab wound injuries, gunshot injuries, and blunt trauma. Patients were divided into 4 groups according to the localisation of the cardiac injury; ventricle, atrium, great vessels and ventricular injury, accompanied by coronary artery injury.

Current ATLS guidelines were used as a guide to determine the indications and contraindications. EDRT was performed under Emergency Department conditions to patients with thoracic trauma, who had no pulse and rhythm, who had out of hospital CPR for less than 5 mins and for unstable patients with hypotensive shock. Pupillary reactivity, motor activity and respiratory effort were used as sign of life when deciding whether to perform EDRT in patients with cardiac arrest. EDRT was not performed in patients with signs of traumatic brain injury or without signs of life.
Astandard thoracotomy set that was kept in the ED resuscitation room was used for the EDRT. Technically, thoracotomy incision was made on the right, left or both sides, depending on the area of injury. An approximately $20-\mathrm{cm}$ long incision was made through the sixth intercostal space, and the ribs were opened with the help of a chest retractor. In patients, who underwent CPR before thoracotomy, internal cardiac massage was done after the thoracotomy incision. If shockable rhythm was obtained, defibrillation was performed with an external defibrillator.

Statistical analysis was performed using IBM SPSS Statistics 26.0 (Chicago, IL). Categorical data were expressed as frequency and percentage and compared using Chi-square test. Continuous parametric data were expressed as mean \pm standard deviation (SD). As a result of the analysis, when the smallest theoretical frequency value was less than 5, Fisher's Exact test result was reported. In larger than $2 \times 2$ cross tables, Fisher-Freeman-Halton Exact test was used as an alternative for tables where the expected value was less than 5 and the number of cells exceeds $20 \%$ of the total number of cells. Fisher-Freeman-Halton Exact test is a generalized version of the Fisher Exact test for RxC tables ${ }^{10}$. A p-value less than 0.05 was considered statistically significant.

\section{RESULTS}

A total of 27 EDRT cases were performed in the specified period. All 27 patients were included in the study. Two (7.4\%) of the patients were females, and 25 (92.6\%) were males. The mean age of the patients was $32.30 \pm 15.25$ (range 14-67) years. In terms of gender, survival was $52 \%$ in men and $50 \%$ in women (Table I). The 6-month survival was $51.9 \%$ and ROSC was achieved in $61.1 \%$ of patients who underwent CPR. No neurological deficit was observed in any of the patients with a 6-month survival. The comparison of the variables in terms of 6-month survival is given in Table I, and the comparison in terms of ROSC is given in Tablell.

When evaluated according to the mechanism of injury, there were six blunt trauma cases, six gunshot cases and 15 stab-wound cases. All blunt trauma and gunshot patients needed CPR. In stab-wound injuries, six (40\%) of the patients required CPR (Table III). In blunt injuries, none of the six patients survived, but two patients (33.3\%) achieved ROSC after EDRT. The survival rate was 33.3\% in gunshot injuries, and the ROSC in gunshot cases was $66.7 \%$. Although the survival rate was $80 \%$ in stab-wound injuries while the ROSC success rate was $83.3 \%$ after EDRT (Table I and II). When the injury mechanism was investigated in terms of survival, the $p$-value was determined as 0.002 (Table I), while the p value was 0.35 (Table II) when the injury mechanism was investigated in terms of ROSC.

When analysis according to the location of the major injury, 13 cases were in the ventricle, two cases were in the atrium, 10 in the great vessels and two were in the coronary artery (left anterior descending artery) for wounds accompanying ventricular injury. Survival was $61.5 \%$ for ventricular injuries, $100 \%$ in atrial injuries, 20\% in great vessel injuries, and $100 \%$ in ventricular injury accompanied by coronary artery injury. 
Table I: Characteristics of the patients undergoing EDRT in terms of survival.

\begin{tabular}{|c|c|c|c|c|}
\hline \multirow{2}{*}{ Variables } & \multirow{2}{*}{ Category } & \multicolumn{3}{|c|}{6 months survival for all patients $(n=27)$} \\
\hline & & Deaths & Survivors & p-value \\
\hline \multicolumn{2}{|l|}{ All patients } & $13(48.1 \%)$ & $14(51.9 \%)$ & \\
\hline \multirow{2}{*}{ Gender } & Male & $12(48 \%)$ & $13(52 \%)$ & \multirow[b]{2}{*}{$>0.999 *$} \\
\hline & Female & $1(50 \%)$ & $1(50 \%)$ & \\
\hline \multirow{3}{*}{ Mechanism of injury } & Blunt & $6(100 \%)$ & $0(0 \%)$ & \multirow{3}{*}{$0.002 * *$} \\
\hline & Gun Shot & $4(66.7 \%)$ & $2(33.3 \%)$ & \\
\hline & Stab-wound & $3(20 \%)$ & $12(80 \%)$ & \\
\hline \multirow{4}{*}{ Location of major injury } & Ventricle & $5(38.5 \%)$ & $8(61.5 \%)$ & \multirow{4}{*}{$0.02 * *$} \\
\hline & Atrium & $0(0 \%)$ & $2(100 \%)$ & \\
\hline & Great vessels & $8(80 \%)$ & $2(20 \%)$ & \\
\hline & Ventricle + Coronary & $0(0 \%)$ & $2(100 \%)$ & \\
\hline \multirow{2}{*}{ Out of hospital CPR } & Necessary & $10(83.3 \%)$ & $2(16.7 \%)$ & \multirow[b]{2}{*}{0.001} \\
\hline & Not-necessary & $3(20 \%)$ & $12(80 \%)$ & \\
\hline \multirow{2}{*}{ Referred to an AC } & Yes & $2(40 \%)$ & $3(60 \%)$ & \multirow{2}{*}{$>0.999 *$} \\
\hline & No & $11(50 \%)$ & $11(50 \%)$ & \\
\hline
\end{tabular}

Table II: Characteristics of the patients undergoing EDRT after CPR in terms of ROSC.

\begin{tabular}{|c|c|c|c|c|}
\hline \multirow[b]{2}{*}{ Variables } & \multirow[b]{2}{*}{ Category } & \multicolumn{3}{|c|}{ ROSC in patients undergoing CPR before EDRT $(n=18)$} \\
\hline & & ROSC (-) & ROSC (+) & p value \\
\hline \multicolumn{2}{|l|}{ All patients } & $7(38.9 \%)$ & $11(61.1 \%)$ & \\
\hline \multirow{2}{*}{ Gender } & Male & $6(37.5 \%)$ & $10(62.5 \%)$ & \multirow[b]{2}{*}{$>0.999 *$} \\
\hline & Female & $1(50 \%)$ & $1(50 \%)$ & \\
\hline \multirow{3}{*}{ Mechanism of injury } & Blunt & $4(66.7 \%)$ & $2(33.3 \%)$ & \multirow{3}{*}{$0.350 * *$} \\
\hline & Gun Shot & $2(33.3 \%)$ & $4(66.7 \%)$ & \\
\hline & Stab-wound & $1(16.7 \%)$ & $5(83.3 \%)$ & \\
\hline \multirow{4}{*}{ Location of major injury } & Ventricle & $2(33.3 \%)$ & $4(66.7 \%)$ & \multirow{4}{*}{$0.868 * *$} \\
\hline & Atrium & $0(0 \%)$ & $1(100 \%)$ & \\
\hline & Great vessels & $5(50 \%)$ & $5(50 \%)$ & \\
\hline & Ventricle + Coronary & $0(0 \%)$ & $1(100 \%)$ & \\
\hline \multirow{2}{*}{ CPR } & Out of hospital & $6(50 \%)$ & $6(50 \%)$ & \multirow{2}{*}{$0.316 *$} \\
\hline & In-hospital & $1(16.7 \%)$ & $5(83.3 \%)$ & \\
\hline
\end{tabular}

Table III: Characteristics of patients undergoing CPR.

\begin{tabular}{|c|c|c|c|c|c|}
\hline \multirow{2}{*}{ Variables } & \multirow{2}{*}{ Category } & \multicolumn{4}{|c|}{ CPR status for all patients $(n=27)$} \\
\hline & & Out of hospital CPR & In-hospital CPR & CPR not performed & p-value \\
\hline \multicolumn{2}{|l|}{ All patients } & $12(44.4 \%)$ & $6(22.2 \%)$ & $9(33.3 \%)$ & \\
\hline \multirow[b]{2}{*}{ Gender } & Male & $10(40 \%)$ & $6(24 \%)$ & $9(36 \%)$ & \multirow[b]{2}{*}{$0.487^{* *}$} \\
\hline & Female & $2(100 \%)$ & $0(0 \%)$ & $0(0 \%)$ & \\
\hline \multirow{3}{*}{ Mechanism of injury } & Blunt & $4(66.7 \%)$ & $2(33.3 \%)$ & $0(0 \%)$ & \multirow{3}{*}{$0.008 * *$} \\
\hline & Gun Shot & $5(83.3 \%)$ & $1(16.7 \%)$ & $0(0 \%)$ & \\
\hline & Stab-wound & $3(20 \%)$ & $3(20 \%)$ & $9(60 \%)$ & \\
\hline \multirow{4}{*}{ Location of major injury } & Ventricle & $5(38.5 \%)$ & $1(7.7 \%)$ & $7(53.8 \%)$ & \multirow{4}{*}{$0.02 * *$} \\
\hline & Atrium & $1(50 \%)$ & $0(0 \%)$ & $1(50 \%)$ & \\
\hline & Great vessels & $6(60 \%)$ & $4(40 \%)$ & $0(0 \%)$ & \\
\hline & Ventricle + Coronary & $0(0 \%)$ & $1(50 \%)$ & $1(50 \%)$ & \\
\hline \multirow{2}{*}{ Referred to an AC } & Yes & $1(20 \%)$ & $2(40 \%)$ & $2(40 \%)$ & \multirow{2}{*}{$0.468 * *$} \\
\hline & No & $11(50 \%)$ & $4(18.2 \%)$ & $7(31.8 \%)$ & \\
\hline
\end{tabular}

When evaluated according to the success of ROSC in patients who needed CPR, the ROSC success rate was $66.7 \%$ in ventricular injuries, $100 \%$ in atrial injuries, $50 \%$ in great vessel injuries and $100 \%$ in coronary artery injuries accompanying ventricle injury (Table I, Table II). Regarding survival, the $p$ value was found to be 0.02 for the risk attributed to the location of major injury (Table I). On the other hand, regarding ROSC, the p-value was found to be 0.868 (Table II).

When the relationship between out of hospital CPR and survival was investigated, the survival was $16.7 \%$ for patients who underwent out of hospital CPR and $80 \%$ for patients who did not need out of hospital CPR ( $p=0.001$, Table I).

ROSC was compared for patients with out of hospital cardiac arrest or in-hospital cardiac arrest. ROSC success was observed in $50 \%$ of patients who needed out of hospital CPR before EDRT, while ROSC success was observed in $83.3 \%$ of patients whose spontaneous circulation was lost in the hospital ( $p=0.316$, Table I).

When evaluated in terms of the need for an advanced 
centre, five patients needed an advanced centre after EDRT and were referred. Survival in these patients was $60 \%$ (Table I). One of these patients received out of hospital CPR and two required in-hospital CPR (Table III).

\section{DISCUSSION}

Although resuscitative thoracotomy was described by Schiff in 1874, Beall et al. published their paper in 1967 in which they argued that thoracotomy should be performed in the ED for resuscitation of patients with penetrating chest trauma. ${ }^{11,12}$ EDRT can be a life-saving procedure when performed by a qualified surgeon in the presence of indications. The principles of EDRT were laid down in 2004 by the American College of Surgeons Committee on Trauma. ${ }^{8}$ Advanced trauma life support (ATLS) emphasised the importance of EDRT on all updates from 2004 onwards.

In cases with cardiac arrest after penetrating and blunt trauma, ATLS 2018 guidelines reported that the EDRT increased survival by a minimum of $10 \%$ at specialised centres. It was recommended that 'emergency resuscitative thoracotomy' should be performed in the ED by qualified thoracic surgeons in patients presenting with post-traumatic arrest. $^{9}$

EDRT indications for penetrating thoracic trauma were determined as suffering cardiac arrest in the ED, confirmed cardiac injury in traumatic arrest patients, hypotensive patients (SBP <60) despite fluid resuscitation, in-hospital traumatic cardiac arrest with documented cardiac activity prior to admission and a maximum of 5 min of out of hospital CPR. EDRT indications for blunt thoracic trauma are defined as witnessed cardiac arrest in ED and rapid bleeding of more than $1500 \mathrm{ml}$ from the chest tube. ${ }^{9}$ Furthermore, 2018 ATLS guideline recommends performing EDRT in cases where spontaneous circulation does not return with $\mathrm{CPR}^{9}$. If CPR time is longer than 5 minutes for blunt trauma and 15 minutes for penetrating trauma, thoracotomy is contraindicated with a stipulation of no signs of life. ${ }^{13}$

Peter et al. analysed the survival rates of emergency thoracotomies over a period of 25 years and reported the survival rates as $1.4 \%$ for blunt injuries, $16.8 \%$ for stab wounds, and $4.3 \%$ for gunshot wounds. ${ }^{14}$ In a meta-analysis published by Seamon et al. in 2015 , survival rate was reported as $10.6 \%$ considering all penetrating thoracic traumas. In the same meta-analysis, survival was reported as $2.3 \%$ for all blunt thoracic traumas. ${ }^{15}$ In a study published in 2020, where 17 cases of EDRT were reported in a trauma centre between 2006 and 2018, 1-year survival was reported only for one patient. $^{16}$

Thoracotomy can be performed in selected cases by experienced surgeons even out of hospital. In a study of 33 cases, who underwent out of hospital emergency thoracotomy, spontaneous circulation was restored in nine patients
(27.2\%). One case survived without neurological damage and was discharged from the hospital. ${ }^{17}$ In this study, one reason explaining the high overall survival rate was that EDRT was performed to patients without allowing cardiac arrest. Although survival is less in out of hospital CPR patients, it is at a significant level (16.7\%). The effect of out of hospital CPR length on EDRT success has been known since $1992 .{ }^{18}$ Studies comparing patients who had out of hospital CPR less than 5 minutes and patients who did not need CPR are not common in the literature. In the current study population, 12 patients needed out of hospital CPR whereas 15 patients did not need out of hospital CPR. While survival was $16.7 \%$ in patients who needed out of hospital CPR, it was $80 \%$ in patients who did not need out of hospital CPR. ROSC success was observed in $50 \%$ of patients with out of hospital CPR and $83.3 \%$ of patients who need in-hospital CPR. These findings demonstrate the negative effect of CPR length on ROSC success and are consistent with the literature.

Generally, for the reported cases survival and ROSC success rates $(51.9 \%$ and $61.1 \%$, respectively) were quite high compared to the studies in the literature. The fact that stab-wound injury cases were in the majority in this study is one explanation for this effect. The authors believe that the application of indications and contraindications with strict rules and not performed EDRT to patients with CPR longer than 5 minutes or to patients with no signs of life were the crucial reasons for achieving these high success rates. Survival is significantly lower $(16.7 \%-80 \%)$ in patients who have had out of hospital CPR, even if CPR was performed for less than 5 minutes compared to those who did not need CPR. In addition, informing the ED during transportation of the patients with severe trauma made fast and early EDRT possible.

When evaluated according to the mechanism of injury, there was no survival in blunt trauma. This finding is consistent with the literature. ${ }^{13,14}$ When the localisation of injury was examined in six patients who underwent EDRT due to blunt trauma, large vessel injuries were observed in all patients. This finding may help explain the high mortality rates in blunt trauma.

Survival was observed in two patients with great vessel injuries, one was injured by a gunshot and the other by a stab-wound. The gunshot case was an injury that occurred inside the hospital. The other stab-wound case occurred in an area close to the hospital. The survival of these two patients with great vessel injuries, who had the chance to receive very early medical intervention, shows that early EDRT increases survival even in great vessel injuries, revealing the importance of early intervention. As a matter of fact, there are countries where out of hospital thoracotomy is started at the scene, and it is obvious that organisations that make early intervention possible should become widespread. 
In this study, stab-wound injuries had a survival rate of $80 \%$, whereas ROSC success was $83.3 \%$ after EDRT. Previous studies have also reported that stab-wound injuries have a higher survival rate after EDRT compared to gunshot and blunt injuries. ${ }^{19}$

EDRT provided time for five patients who needed treatment at an advanced centre and resulted in the survival of three of them.

The hospital where the study was conducted is a secondary hospital of a county with a population of 155,000 . This hospital is not a trauma centre with extensive facilities, and it is approximately $1.5 \mathrm{~h}$ away from the nearest tertiary hospital by ambulance. Although it is not a trauma hospital, it was organised in a manner similar to a trauma team in terms of early intervention for patients who may have an indication for EDRT. Patients in need of cardiovascular surgery are referred after the primary intervention, and EDRT is important in terms of saving time for the patients. It is also important that this study was a successful exception to the de-facto acceptance that EDRT can only be done in trauma centres.

In this study, one patient suffered hypoxic brain injury. He survived for 20 days. After the decision of brain death, the life guardians provided the consent for organ donation. In addition to being a life-saving intervention, EDRT gives the life guardians time to assess the need for organ donation when death is unavoidable. Schrüniger et al. published a study in 2010 and reported that 11 of 263 patients who underwent EDRT were potential organ donors due to brain death. ${ }^{20}$

The mechanism with the highest survival after EDRT is stab-wound injuries. When analysed according to injury localisation, the survival rate was found highest in patients with a ventricular injury. Survival was observed to be higher in those who did not require out of hospital CPR. Training programmes of clinicians, especially emergency specialists', should be organised in order to disseminate the EDRT practice. It should be kept in mind that EDRT will increase survival in penetrating injuries, especially in stab-wounds.

The fact that the data is obtained from the records and the planning cannot be done, is an important disadvantage of retrospective studies. The number of cases may not be very high in diseases or interventions with rare incidence.

\section{CONCLUSION}

With the dissemination of training for EDRT and with encouragement that it can be performed in smaller hospitals without trauma centres, EDRT will be life-saving both directly and indirectly by increasing the number of organ donors. It should not be forgotten that early and rapid intervention will be life-saving.
Ethical Approval: This study was carried out in accordance with the ethical principles of the Declaration of Helsinki and was approved by the Ethics Committee of Balıkesir University (Decision Number: 2020/52, Decision Date: 8 April 2020).

\section{PATIENTS' CONSENT:}

In this retrospective study, the data were collected from the HIMS database after the approval of the Ethics Committee.

\section{CONFLICT OF INTEREST:}

The authors declared no conflict of interest.

\section{AUTHORS' CONTRIBUTION:}

FD: Substantial contributions to the conception or design of the work; the acquisition, analysis, or interpretation of data for the work; drafting the work or revising it critically for important intellectual content; accepted the final approval of the version to be published; accepted to be accountable for all aspects of the work in ensuring that questions related to the accuracy or integrity of any part of the work are appropriately investigated and resolved.

FG: Acquisition, analysis, or interpretation of data for the work; accepted the final approval of the version to be published; accepted to be accountable for all aspects of the work in ensuring that questions related to the accuracy or integrity of any part of the work are appropriately investigated and resolved.

\section{REFERENCES}

1. Organization WH. World health statistics 2019: Monitoring health for the SDGs, sustainable development goals. 2019.

2. Härgestam M, Lindkvist M, Jacobsson M. Brulin C, Hultin M. Trauma teams and time to early management during in situ trauma team training. BMJ Open 2016; 6(1):e009911. doi: 10.1136/bmjopen-2015-009911.

3. Dutton R. Current concepts in hemorrhagic shock. Anesthesiol Clin 2007; 25(1):23-34. doi: 10.1016/j.atc.2006.11.007.

4. MacKenzie EJ, Rivara FP, Jurkovich GJ, Nathens AB, Frey KP, Gleston BL, et al. A national evaluation of the effect of trauma-center care on mortality. $N$ Engl J Med 2006; 354(4): 366-78. doi: 10.1056/NEJMsa052049.

5. Demetriades D, Murray J, Charalambides K, Alo K, Velmahos $G$, Rhee $P$, et al. Trauma fatalities: Time and location of hospital deaths. J Am Coll Surg 2004; 198(1):20-6. doi: 10.1016/j.jamcollsurg.2003.09.003.

6. Levitsky S. New insights in cardiac trauma. Surg Clin North Am 1975; 55(1):43-55. doi: 10.1016/s0039-6109(16) 40529-3.

7. Levitsky S, James PM, Anderson RW, Hardaway RW. Vascular trauma in Vietnam battle casualties: An analysis of 55 consecutive cases. Ann Surg 1968; 168(5):831-6. doi: 10.1097/00000658-196811000-00008.

8. Manual A. Advanced trauma life support for doctors. 2004; 1:7.

9. Henry S. ATLS 10th edition offers new insights into managing trauma patients. 2018.

10. Freeman $\mathrm{GH}$, Halton $\mathrm{JH}$. Note on an exact treatment of 
contingency, goodness of fit and other problems of significance. Biometrika 1951; 38(1-2): 141-9.

11. Beall Jr A, Diethrich E, Cooley D, DeBakey ME. Surgical management of penetrating cardiovascular trauma. South Med J 1967; 60(7):698-704. doi: 10.1097/00007611196707000-00005.

12. Burlew CC, Moore EE, Moore FA, Coimbra R, McIntyre Jr RC, Davis JW, et al. Western trauma association critical decisions in trauma: Resuscitative thoracotomy. Trauma Acute Care Surg 2012; 73(6):1359-63. doi: 10.1097/TA.0b01 $3 e 318270 \mathrm{~d} 2 \mathrm{df}$.

13. Aseni P, Rizzetto F, Grande AM, Bini R, Sammartano F, Vezzulli $F$, et al. Emergency Department Resuscitative Thoracotomy: Indications, surgical procedure and outcome. A narrative review. Am J Surg 2021; 221(5):1082-95. doi: 10.1016/j.amjsurg.2020.09.038.

14. Rhee PM, Acosta J, Bridgeman A, Wang D, N Rich MJ. Survival after emergency department thoracotomy: review of published data from the past 25 years. J Am Coll Surg 2000; 190(3):288-98. doi: 10.1016/s1072-7515(99)00 233-1.

15. Seamon MJ, Haut ER, Van Arendonk K, Barbosa R, Chiu W, Dente $C$, et al. An evidence-based approach to patient selection for emergency department thoracotomy: A prac- tice management guideline from the Eastern Association for the Surgery of Trauma. J Trauma Acute Surg 2015; 79(1):159-73. doi: 10.1097/TA.0000000000000648.

16. Thorsen K, Vetrhus M, Narvestad J, Reite A, E Tjosevik K, Søreide $K$, et al. Performance and outcome evaluation of emergency resuscitative thoracotomy in a Norwegian trauma centre: A population-based consecutive series with survival benefits. Injury 2020; 51(9):1956-60. doi: 10.1016/ j.injury.2020.05.040.

17. Van Vledder MG, Van Waes OJ, Kooij FO, Peters J, M Van Lieshout E, H J Verhofstad M. Out of hospital thoracotomy for cardiac arrest after penetrating thoracic trauma. Injury 2017; 48(9):1865-69. doi: 10.1016/j.injury.2017.04.002.

18. Durham 3rd L, Richardson RJ, Wall Jr MJ, Pepe PE, Mattox $\mathrm{KL}$. Emergency center thoracotomy: Impact of prehospital resuscitation. J Trauma 1992; 32(6):775-9. doi: 10.1097/ 00005373-199206000-00019.

19. Lustenberger T, Labler L, Stover J, B Keel MJ. Resuscitative emergency thoracotomy in a Swiss trauma centre. Br J Surj 2012; 99(4):541-8. doi: 10.1002/bjs. 7706.

20. Schnüriger B, Inaba K, Branco BC, Salim A, Russell K, Lam L, et al. Organ donation: An important outcome after resuscitative thoracotomy. J Am Coll Surg 2010; 211(4):450-5. doi: 10.1016/j.jamcollsurg.2010.06.012. 